\title{
Facial clefts diagnosed before birth: routine ultrasound screening at the multi-disciplinary center for prenatal diagnosis (PCPD)
}

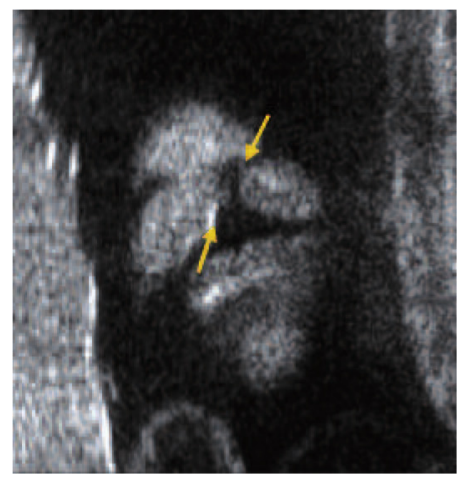

\author{
Pascale BACH-SÉGURA
}

\begin{abstract}
\section{KEY WORDS}

Facial cleft,

Prenatal diagnosis,

Ultrasound.
\end{abstract}

Since 2005 in France, the national technical committee of prenatal ultra-sound screening (CNTE) has recommended that pregnant women have a routine second trimester ultrasound screening for facial clefts. The view on the coronal reference plane visualizes the continuity of the upper lip, and makes it possible to detect most facial clefts, but not isolated palatal clefts.

\section{1 - INTRODUCTION}

Embryo-fetal malformations occur in approximately $3.5 \%$ of all births $\left({ }^{*}\right)$. Clefts, all types combined, represent nearly $4 \%$ of these malformations and roughly 1 to 1.3 per 1000 births with geographic and ethnic variation.

The variable appearance of facial clefts corresponds to very different pathological 
entities. Labial clefts with or without a palate cleft are isolated in 2/3 of the cases whereas a cleft palate without cleft lip, rarely detected by prenatal ultrasound, is more frequently associated with a genetic syndrome ${ }^{3}$. When a midline cleft is detected, examiners search for anomalies related to the central nervous system. More than 300 syndromes including a facial cleft have been identified. Clefts therefore represent a very heterogeneous group of malformations in terms of phenotype and genotype and are thus classified as a rare disease according to the Orphanet database.

According to data in the Alsace Registry of congenital malformations, collected between 1995 and 2006, $52 \%$ of clefts have been detected by ultrasound before birth. The requirement that sonographers participating in prenatal screening receive specialized training, the application of recommendations of the national technical committee of prenatal ultrasound screening (CNTE) in place since 2005 and technological advances in ultrasound equipment explain the increase in the rate of detection compared to data in the Registries prior ${ }^{2}$ to 2000.

Until 2005, a prenatal examination required private consultation with an expert. Now, it is done routinely even when screening what are presumed to be low-risk pregnancies. The detection procedure is based on the acquisition of a reverse face frontal view of the lower part of the face using ultrasound during the second trimester of pregnancy. This view (Fig. 1) allows sonographers to visualize the continuity of the upper lip and thus makes it possible to detect facial clefts except for isolated palatal clefts.

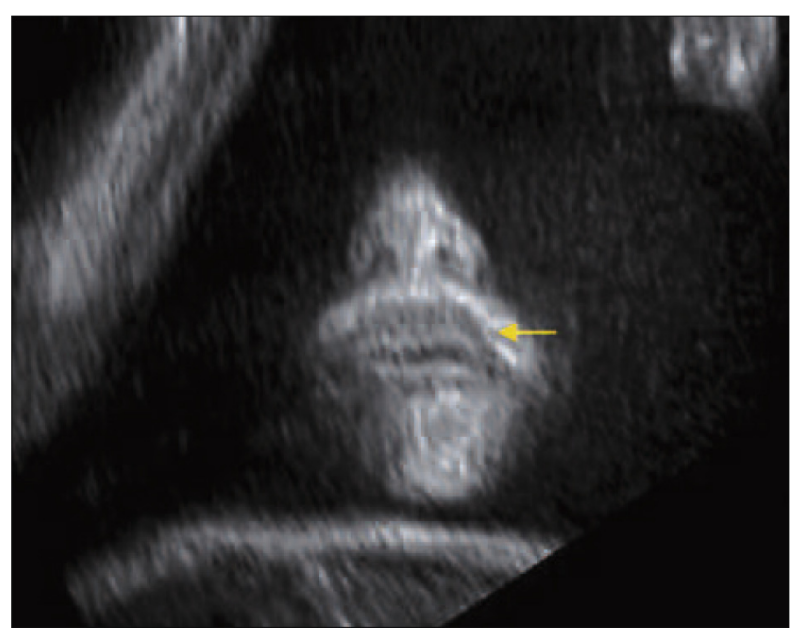

Figure 1

Normal appearance at 22 weeks.

The view of the face on the coronal reference plane is recommended by the CNTE (incidence nose/mouth/ chin). The normal continuity of the upper lip is visible, the nasal orifices are symmetrical.

Prenatal diagnosis of a congenital fetal malformation has as its goal:

- To begin the multi-disciplinary management and care of a potentially curable pathology before birth. For malformations such as labial clefts or lip/palate clefts that involve no immediate risk to life and that can be operated on during the neonatal period, the impact of the prenatal diagnosis can be beneficial and can help the parents prepare to welcome their future child by meeting the pediatricians and the surgeons and by facilitating a better integration of the child into the family, society and school. On the other hand, the impact of the diagnosis can be negative and can generate intense anxiety for some parents;

$\left({ }^{*}\right)$ Children born alive or stillborn starting from the $22^{\text {th }}$ week of gestation. 
- To detect a particularly serious incurable anomaly, on account of which ending the pregnancy for medical reasons is possible even at the end of the third trimester, based on the current regulatory policy in France.

\section{2 - MODALITIES OF MEDICAL SURVEILLANCE OF PREGNANCY BY ULTRASOUND}

The technical quality of ultrasound screening depends on several different factors such as: fetal presentation on the viewing screen, uterine crowding (gestational age, the status of the liquid in the intra-amniotic environment, twin pregnancy) and acoustic factors involving the parietal walls of the uterus.

The detection of a fetal anomaly is most often fortuitous during testing with ultrasound of a pregnancy presumed to be low risk. Sometimes, the examination is immediately focused in search of a specific anomaly due to increased risk because of: previous pregnancy anomalies, advanced age, pathology, immune-depression, biological markers, exposure to exogenous factors, an ultrasound warning sign previously detected.

Finally, a second ultrasound may be required if there are technical difficulties that make it impossible to complete the initial screening exam.

\section{- Ultrasound screening tests}

Ultrasound screening that is routinely offered to patients during pregnancy is not a mandatory medical exam. The patient has to be systematically informed of the goals and limits associated with this procedure and consent before the physician can write a prescription. This screening exam is performed so that the obstetrician can closely monitor the preg- nancy in the course of each of the trimesters, between the 11th and 14th weeks of gestation, the 20th and 25th weeks and the 30th and 35th weeks. These tests are three of the four obstetrical ultrasounds that are performed and each trimester screening corresponds to precise testing objectives.

According to recommendations of the CNTE elaborated in 2005, the second semester (22-24 weeks of pregnancy) is an essential time for analyzing the face and performing a screening for morphological abnormalities of the fetus. The continuity of the upper lip must be visualized on the frontal view, which tangentially borders the lower part of the face.

Visualization of a cleft lip is relatively easy after the 20 week of pregnancy: revealing a break in the continuity of the upper lip with a view on the coronal reference plane (Figs. 2 and $3)$, whereas an isolated palatal cleft is only rarely detected because of the concave shape of the palate and its suboptimal position with acoustic shadowing of osseous mineralization in the maxillary midface ${ }^{9}$.

Sometimes, screening for cleft lip/ palate is possible at the beginning of the 3rd trimester of pregnancy when the fetal face is accessible by using transvaginal ultrasound ${ }^{6}$. 


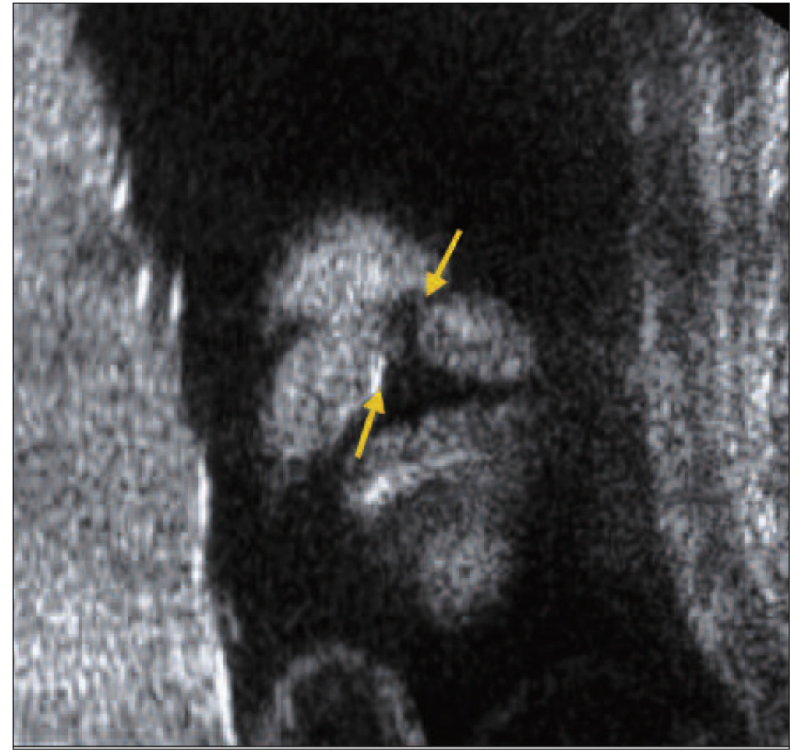

Figure 2

Left unilateral cleft lip (24th week).

Sequence of the coronal view of the face. Break in the continuity of the upper lip.

When a cleft is identified or when the analysis of a case is difficult, the patient receives a prescription for a second ultrasound for diagnostic purposes. This testing procedure, applicable to any situation that involves a presumed pathology, makes the sonographer (physician or mid-wife) deal with the difficult task of breaking the news to the future parents. After they have had time to adjust to the news, the future parents must be given a detailed explanation. It is essential to make a precise diagnosis and to confirm the absence of any genetic syndrome by referring them to a sonographer who will be responsible for sending their records to the center for multi-disciplinary prenatal diagnosis. In the case of a cleft lip or cleft lip/

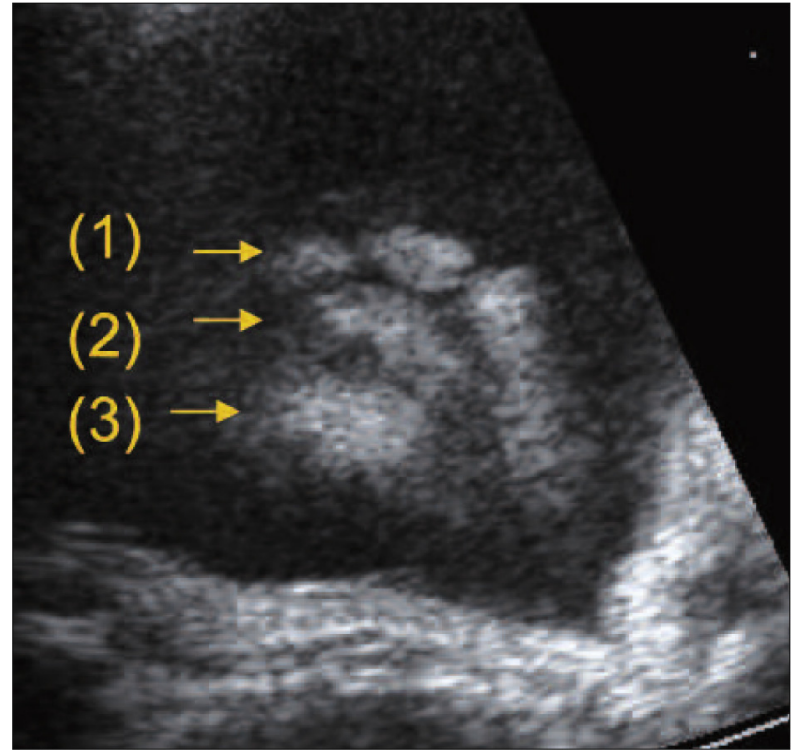

Figure 3

Bilateral cleft lip (23th week).

Coronal view of the chin/mouth:

(1) 2 breaks in the continuity of the upper lip are visible;

(2) lower lip;

(3) chin.

palate, the largely isolated aspect of these types of cleft is emphasized.

Undoubtedly, when a couple plans a pregnancy and decides to become parents, they probably have certain expectations. From this perspective, the future parents are ultimately stunned by the sudden and unexpected bad news.

\section{- The follow-up ultrasound or "ultrasound to ultrasound com- parison"}

Therefore, the follow-up ultrasound provides specific details about the fetal pathology, the severity of the cleft as well as practical guidance for the parents.

In our practice, the follow-up ultrasound is frequently performed around the 26th week and is planned several 


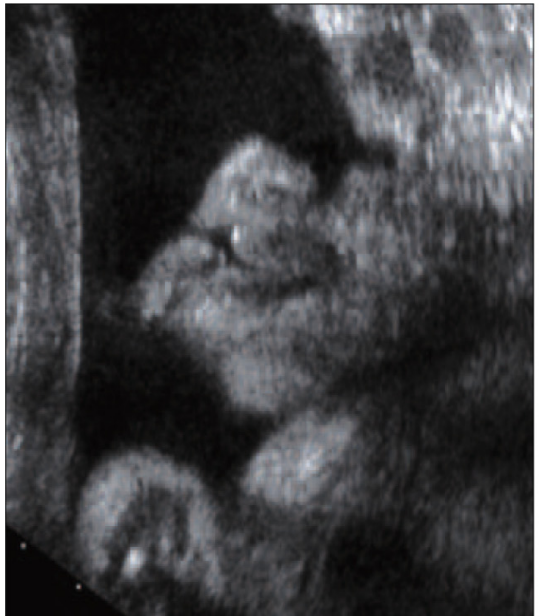

a

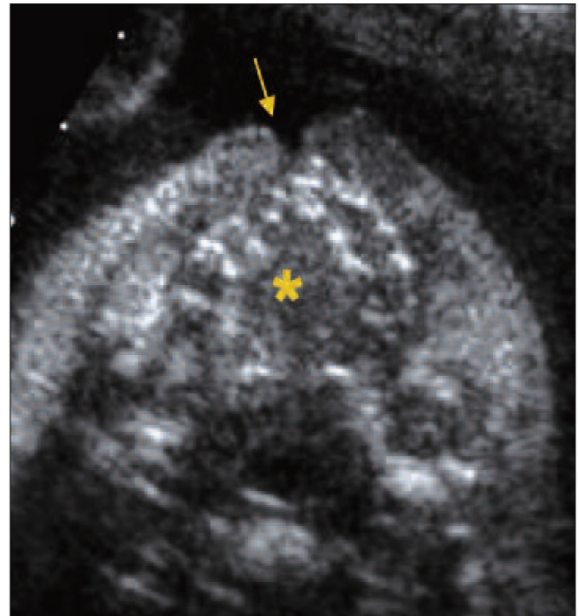

b

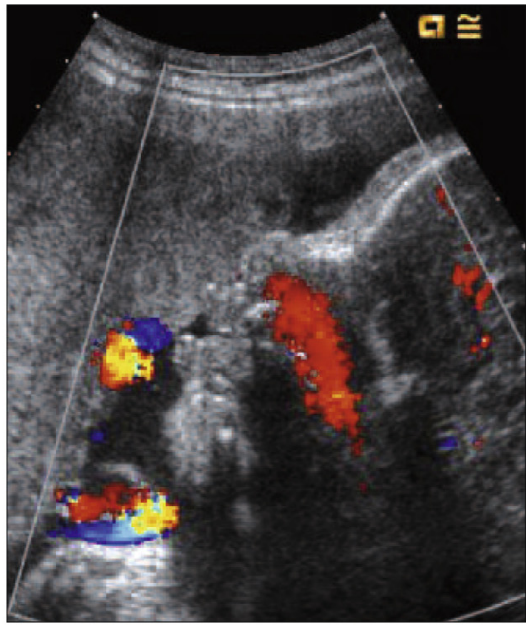

C

Figures $4 a$ to $4 c$

Right labio-alveolar cleft (26th week of pregnancy):

a: coronal view of the face: break in the continuity of the upper lip with nostril asymmetry, deviation of the nasal pyramid to the left;

b: axial view of the face showing the alveolar arch of the upper jaw: disharmonious alveolar arch with respect to the labial defect (arrow). The angle of the view makes it possible to see the entire hard palate (*) from behind;

c: sagittal view, there is no evidence of abnormal passage between the nasal cavities and the mouth cavity with color Doppler imaging.

weeks after delivering the unexpected bad news. This two to three week interval facilitates the exchange between the practitioner and the couple and allows the future parents to more easily understand all the information concerning this pathology. Obviously, the interval can justifiably be shorter if parental stress is too great.

During this follow-up examination, some time is spent assessing the cleft lip and palatal lesions: right or left laterality or bilaterality, the symmetry of the nasal orifices is clearly brought into focus from a surfacerendered three-dimensional frontal view of the face; the width of the defect can also be measured. The integrity of the alveolar ridge and of the hard palate is examined from axial views (Figs. 4 and 5).

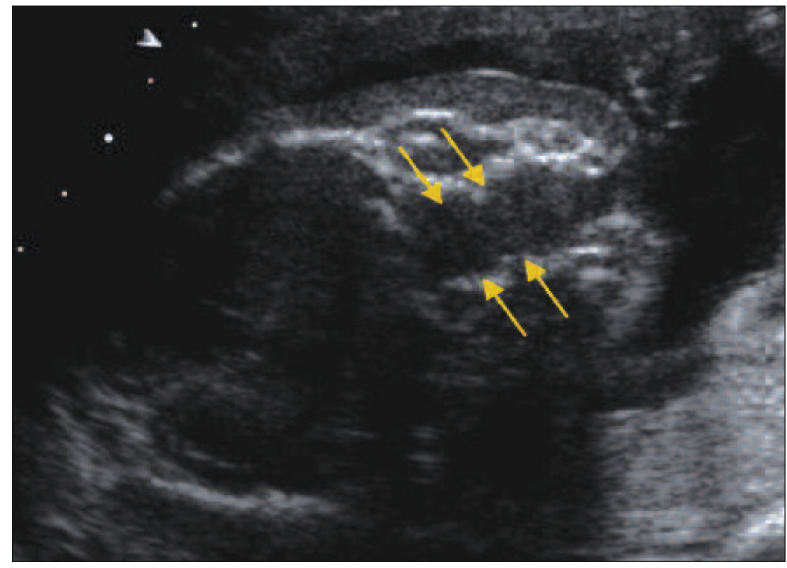

Figure 5

Complete midline cleft (23th week of pregnancy). Axial view of the upper jaw. A wide cleft palate (arrows) beneath labio-alveolar cleft.

The anterior section of the saddlenose, the philtrum and the chin are visualized from a mid-sagittal view of 


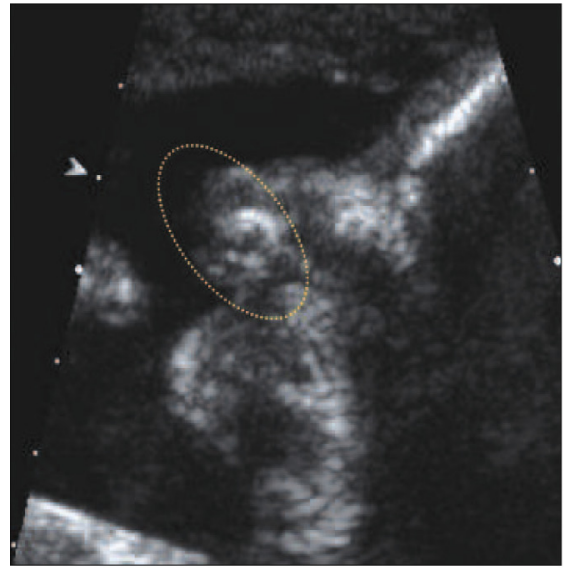

a

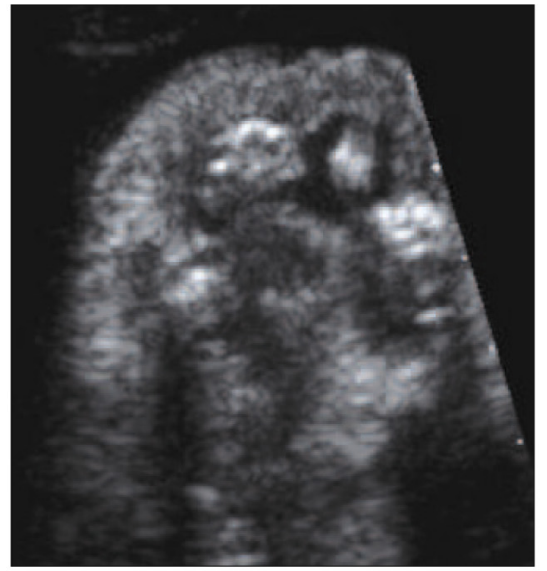

b

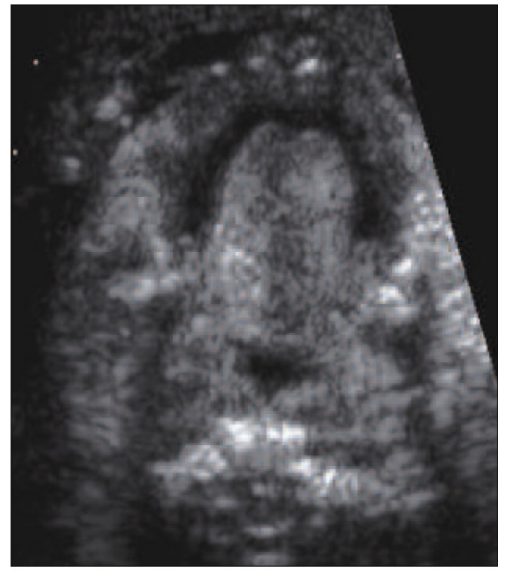

C

Figures 6a to 6c

Complete bilateral cleft (25th week of pregnancy):

a: midline sagittal view: facial bud in the maxillo-labial area (circled) and visible under the tip of the nose;

$b$ : axial view of the face showing the alveolar ridge of the upper jaw: the two clefts of the maxilla extend beyond the hard palate. Tooth buds are visible within the facial bud;

c: axial view of the face above the alveolar ridge of the upper jaw: high tongue position because of the wide cleft palate.

the face, the profile is abnormal when there is a bilateral cleft with protrusion of a midline bud (Fig. 6).

When a cleft palate is wide enough, a sagittal and/or paramedian view of the face makes it possible to see the protrusion of the tongue into the nasal fossae during movements involved in swallowing. The cleft is sometimes visible on this same view (Fig. 7).

Doppler color imaging makes it possible to visualize nasal and amniotic flow and therefore sometimes to detect abnormal flow between the oral and the nasal cavities ${ }^{1}$. This imaging is more detailed after the 28th week of gestation and, after the mother provides an increased carbohydrate supplement now that nasal and amniotic flow are physiologically more frequent.

Another essential element of follow-up ultrasound is to look for other manifestations of genetic abnormalities that are associated with a cleft. The test specifically focuses on the brain, the heart, the kidneys, the ears, the extremities and the perineum. An examination of the fetal contours is equally informative in cases of atypical clefts. Identifying areas of constriction and amputation can indicate amniotic band syndrome.

Finally, the technician may at times suggest that the couple explore additional imaging techniques:

- In the event of a midline cleft or bilateral cleft with a median hypoplastic facial bud, Multiple Resonance Imaging makes it possible to analyze the inter-hemispheric fissure of the fetal brain. This analysis of the fetal brain is focused on eliminating risk factors associated with lobar holoprosencephalia in order to exclude this anomaly: 

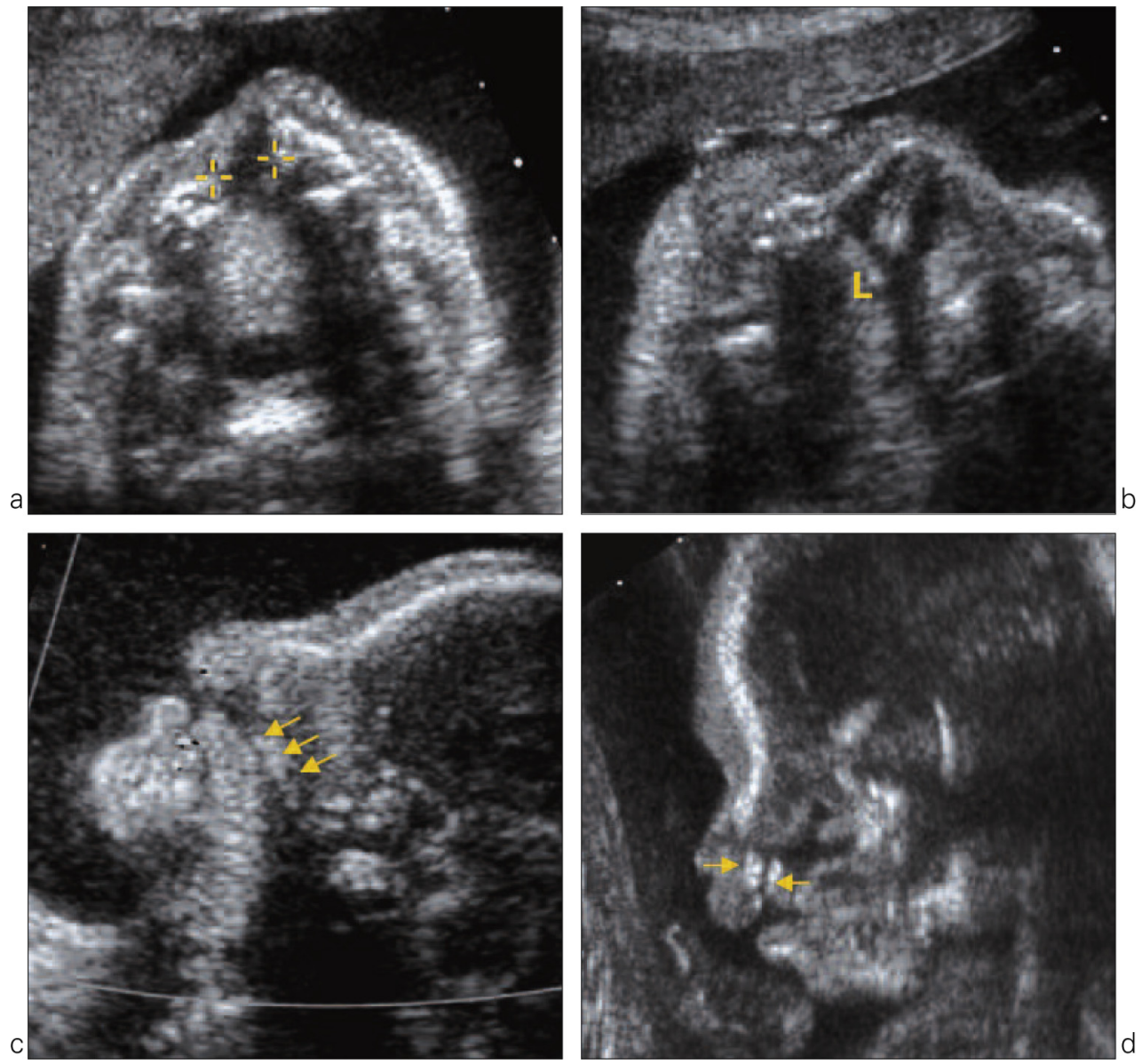

Figures $7 a$ to $7 d$

Right cleft lip/palate (25th week of pregnancy):

a: axial view of the maxillary alveolar ridge: between maxillary diastasis and alveolar gap;

b: axial view of the nasal fossae: protrusion of the tongue into the right nasal fossa;

c: right parasagittal view: only the posterior part of the hard palate is visible (yellow arrows);

$d$ : right parasagittal view going through the cleft palate (yellow arrows): passage between the mouth and the right nostril.

integrity of the falx cerebri (scythe of the brain) without hemispheric separation, lack of fusion of the frontal lobes. This diagnosis, whose prognosis is grim, is not feasible with ultrasound;

- Using fetal tomodensitometry $\left({ }^{*}\right)$ (scanner) makes it possible to visualize the parts of the fetal skeleton that are not clearly visible with ultrasound: axial skeleton or semi-circular canals. This technology is particularly useful when a C.H.A.R.G.E type of syndromic association is suspected $\left({ }^{*}\right)$. The radiation that is delivered is very weak and controlled; the protocols are adapted to the examination of the fetus in utero.

From a diagnostic point of view, 3D ultrasound does not provide better enhancement for detection than other 

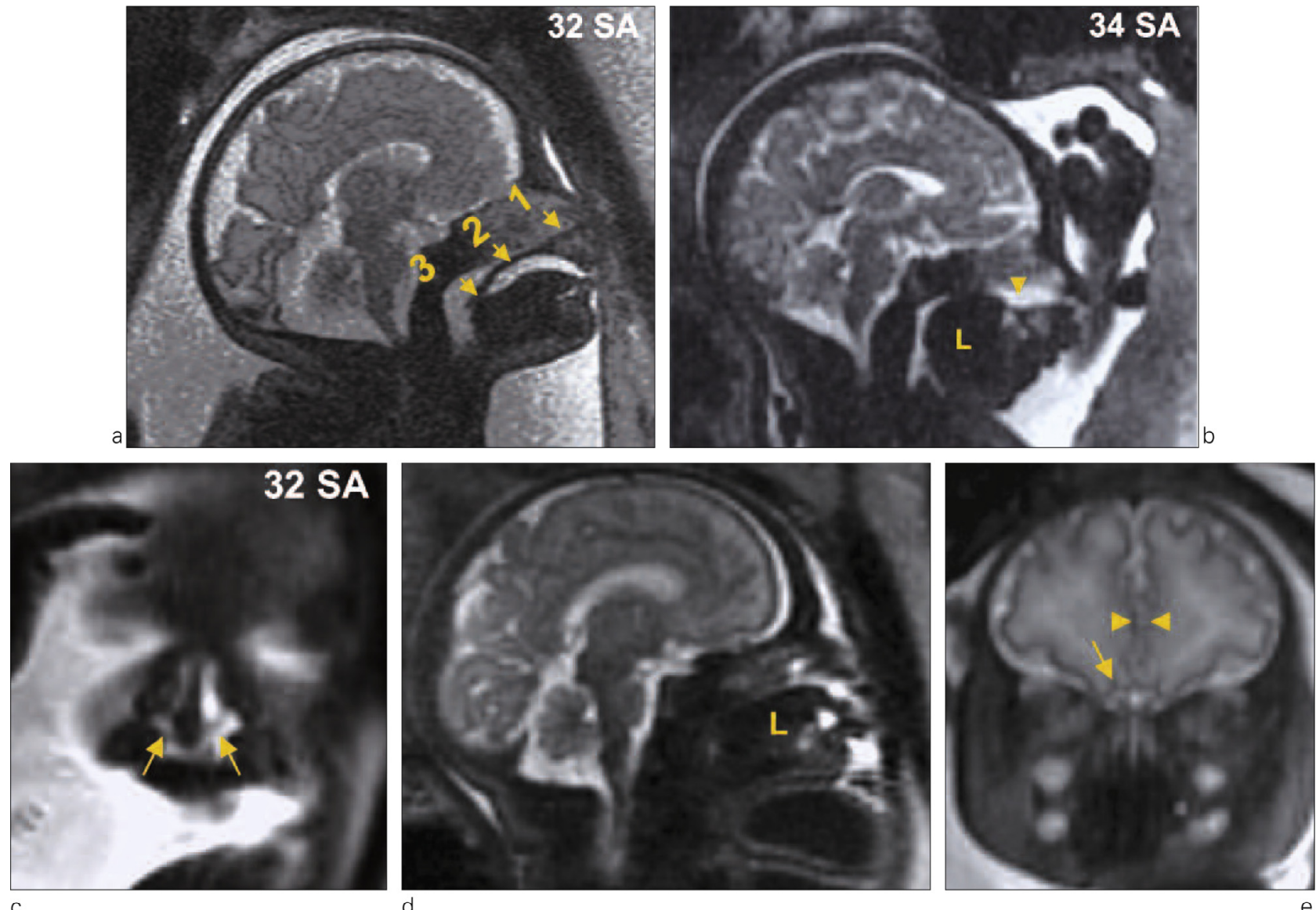

d

e

Figures 8 a to 8 e

Examination of the palate and the fetal brain:

a: fetus witness: midline sagittal view of the face and the brain: (1) primary hard palate, (2) secondary hard palate and (3) soft palate.

b: fetus carrier of the gene for Pierre Robin syndrome: midline sagittal view: glossoptosis (L), interruption of the secondary palate (arrow) whereas the primary palate is quite visible in the foreground. Note the micro-retrognathia; $c$ to e: fetus with bilateral cleft, from left to right:

- coronal view of the face: hard palate not visible (yellow arrows), the mouth connects with the nasal fossae;)

- midline sagittal view: hard palate not visible above the tongue (L) (compare with the witness);

- coronal view of the face seen through the rhinoencephalon: the olfactory bulb is under the olfactory tract lyellow arrow).

Normal appearance of the inter-hemispheric fissure, absence of the fusion of the frontal lobes.

All the above observations make it possible to eliminate lobar holoprosencephalia.

techniques. Therefore, 3D ultrasound is not automatically offered in our center. The realism and precision of 3D images are greeted with mixed reactions by the future parents. We do not want them to live through the shock parents experienced in the past before the advent of prenatal screening as they discovered their child had a cleft at the moment of birth.

Presentations by the doctor of before and after surgery photos of 


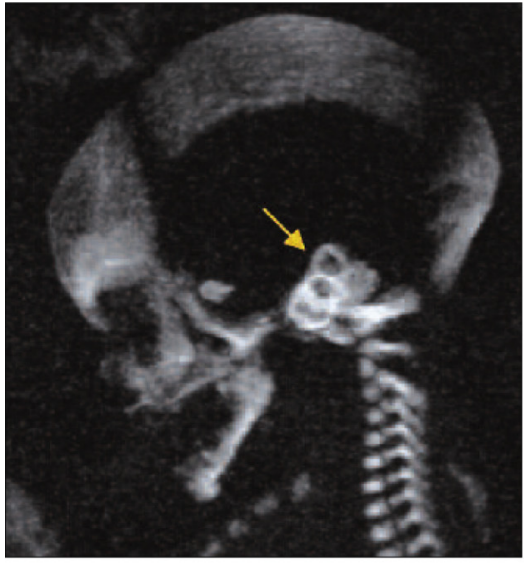

a

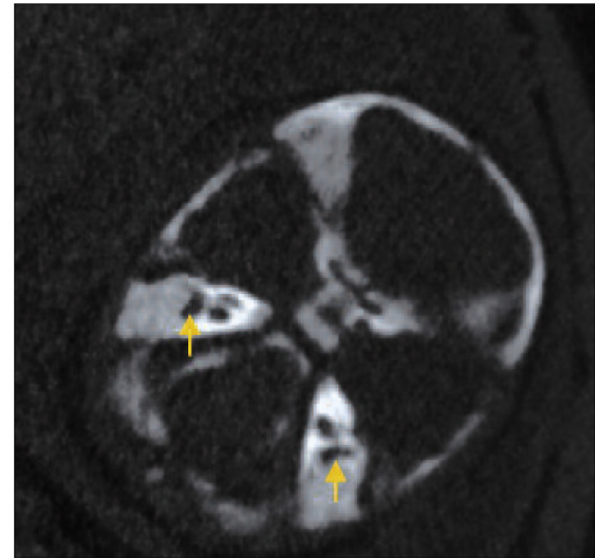

b

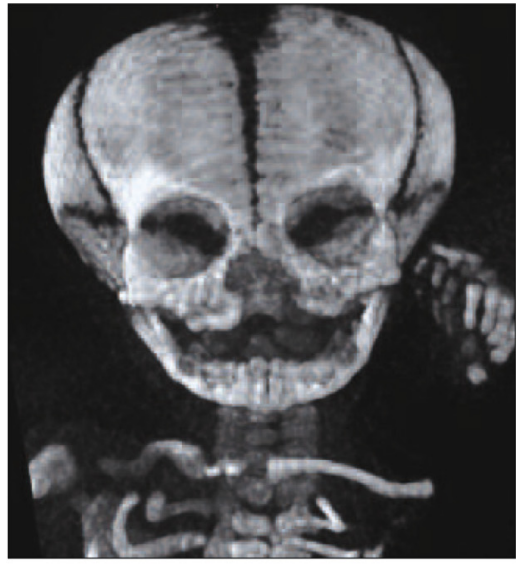

C

Figures 9a to $9 c$

Examination of the midface bones and of the temporal bones of the fetal skeleton with a multi-slice CT scanner: a: witness fetus at 32 weeks of pregnancy. Reconstruction with a thick-slice scan of the semi-circular canals (arrow): the upper two are visible as well as the homolateral lateral in this reconstruction;

b: same fetus. Reconstruction with a thick section view on the oral cavity (arrows) visible behind the cochleae. Interruption of the secondary palate (arrow) whereas the primary palate is very visible in the foreground. Note the micro-retrognathia.

c: fetus with a midline cleft associated with cerebral anomalies. The wide defect in the upper jaw and in the palate is plainly visible.

infants with this defect, along with detailed explanations seem to us to be more appropriate.

At the end of the consultation, the diagnosis of a cleft has been confirmed, explained and specifically identified in terms of type and laterality. The presumption is that the cleft is isolated or on the contrary, associated with abnormalities that make the diagnosis more serious.

The future parents are reminded that the sonographic appearance of a cleft can possibly underestimate its severity compared to the actual postnatal anatomical appearance they will see eventually; therefore, a totally reliable assessment of the cleft defect cannot be made until after the birth of the child.

At the end of this consultation, the parents sign a consent form to have their records forwarded to the multidisciplinary center for prenatal diagnosis (CPDP). They give their consent in order to coordinate a prenatal consultation to do genetic testing and to discuss maxilla-facial surgery. The importance of doing this etiological investigation, of examining the medical history of the family and if need be of determining the fetal caryotype is explained to the couple. Questions concerning the different possible modalities of post-natal treatment will be addressed directly to the surgeon.

${ }^{*}$ ) Administered dose $=$ an adequate dose between 3 and 7 milligrays or the equivalent of two standard frames of the abdomen.

(**) C.H.A.R.G.E. : Colomba, Heart defect, Atresia choanae, growth Restriction, Genital hypoplasia, Ear. 
The prospect of surgically repairing this defect, is always met with approval since it offers a positive outlook to parents who no longer have an image of a "perfect child" after they have seen the ultrasound imaging.

\section{$3-$ CONCLUSION}

In two thirds of the cases, cleft lip and cleft lip/palate are isolated; however, a complete morphological evaluation, including a genetic investigation and sometimes a determination of caryotype, is necessary to confirm the isolation.

The first words used to communicate the bad news are all important. In order to prevent the prenatal detection of a cleft from negatively interfering with the future parents commitment to the pregnancy and dissuade them from making the decision to end it, which still occurs in a very small number of cases, the future parents must receive adequate supportive care and attention that is adapted to their needs and is continued for the duration of the pregnancy.

The quality of this support is based upon a multi-disciplinary collaboration of the medical team that continues from the prenatal period to the birth and extends to the end of the growth period.

\section{REFERENCES}

1. Aubry MC, Aubry JP. Prenatal diagnosis of cleft palate: contribution of color Doppler ultrasound. Ultrasound Obstet Gynecol 1992;2(3):221-4.

2. Clementi M, Tenconi R, Bianchi F, Stoll $C$. Evaluation of prenatal diagnosis of cleft lip with or without cleft palate by ultrasound: experience of 20 European registries. Euroscan Study Group. Prenat Diagn 2000;20(11):8705.

3. Gillham JC, Anand S, Bullen PJ. Antenatal detection of cleft lip with or without cleft palate: incidence of associated chromosomal and structural anomalies. Ultrasound Obstet Gynecol 2009;34(4):410-5.

4. Mailáth-Pokorny M, Worda C, Krampl-Bettelheim E, Watzinger F, Brugger PC, Prayer D. What does magnetic resonance imaging add to the prenatal ultrasound diagnosis of facial clefts? Ultrasound Obstet Gynecol 2010;36(4):445-51.

5. Pannier E, Aubry MC, Viot G, Adamsbaum C, Grangé G, Tsatsaris V, Cabrol D. Que rechercher devant une fente labio-palatine en antenatal ? $2{ }^{\mathrm{e}}$ journée de médecine fœtale de St-Vincent-de-Paul, Port-Royal, mai 2005.

6. Picone $\mathrm{O}$, de Keersmaecker $\mathrm{B}$, Ville $\mathrm{Y}$. Ultrasonographic features of orofacial clefts at first trimester of pregnancy: report of two cases. J Gynecol Obstet Biol Reprod (Paris). 2003;32(8 Pt 1):736-9.

7. Rosen H, Chiou GJ, Stoler JM, Mulliken JB, Tarui T, Meara JG, Estroff JA. Magnetic Resonance imaging for detection of brain abnormalities in fetuses with cleft lip and/or cleft palate. Cleft Palate Craniofac J 2011;48(5):619-22.

8. Sureau C, Henrion R. Rapport du Comité nationale technique de l'échographie de dépistage prénatal. Paris : La Documentation Française, 2005.

9. Wilhem L, Borgers B. The 'equals sign': a novel marker in the diagnosis of fetal isolated cleft palate. Ultrasound Obstet Gynecol 2010;36(4):439-44. 